\title{
A branch and bound algorithm for the global optimization of Hessian Lipschitz continuous functions
}

JM Fowkes, NIM Gould, CL Farmer

November 2011 


\section{C2011 Science and Technology Facilities Council}

Enquiries about copyright, reproduction and requests for additional copies of this report should be addressed to:

RAL Library

STFC Rutherford Appleton Laboratory

R61

Harwell Oxford

Didcot

OX11 0QX

Tel: $+44(0) 1235445384$

Fax: $+44(0) 1235446403$

email: libraryral@stfc.ac.uk

Science and Technology Facilities Council reports are available online at: http:// epubs.stfc.ac.uk

ISSN 1358- 6254

Neither the Council nor the Laboratory accept any responsibility for loss or damage arising from the use of information contained in any of their reports or in any communication about their tests or investigations. 


\title{
A Branch and Bound Algorithm for the Global Optimization of Hessian Lipschitz Continuous Functions
}

\author{
Jaroslav M. Fowkes ${ }^{1,2}$, Nicholas I. M. Gould ${ }^{3,4}$ and Chris L. Farmer ${ }^{5}$
}

\begin{abstract}
We present a branch and bound algorithm for the global optimization of a twice differentiable nonconvex objective function with a Lipschitz continuous Hessian over a compact, convex set. The algorithm is based on applying cubic regularisation techniques to the objective function within an overlapping branch and bound algorithm for convex constrained global optimization. Unlike other branch and bound algorithms, lower bounds are obtained via nonconvex underestimators of the function. For a numerical example, we apply the proposed branch and bound algorithm to radial basis function approximations.
\end{abstract}

1 School of Mathematics, The King's Buildings, University of Edinburgh, EH9 3JZ, Scotland, EU. Email: jaroslav.fowkes@ed.ac.uk .

Current reports available from "http://www.maths.ed.ac.uk/ERGO/preprints.html".

2 This research was supported through an EPSRC Industrial CASE studentship at the University of Oxford in conjunction with Schlumberger.

${ }^{3}$ Computational Science and Engineering Department, Rutherford Appleton Laboratory, Chilton, Oxfordshire, OX11 0QX, England, EU.

Email: nick.gould@stfc.ac.uk .

Current reports available from "http://www.numerical.rl.ac.uk/reports/reports.shtml".

4 This work was supported by the EPSRC grants EP/E053351/1, EP/F005369/1 and EP/I013067/1.

${ }^{5}$ Mathematical Institute, 24-29 St Giles', University of Oxford, OX1 3LB, England, EU. Email: Chris.Farmer@maths.ox.ac.uk .

Computational Science and Engineering Department

Atlas Centre

Rutherford Appleton Laboratory

Oxfordshire OX11 0QX

1st November 2011 


\section{Introduction}

In this paper, we are interested in solving the global optimization problem

$$
\min _{x \in \mathcal{D}} f(x)
$$

where $\mathcal{D} \subset \mathbb{R}^{n}$ is a compact, convex set and $f: \mathcal{C} \rightarrow \mathbb{R}$ is a twice-Lipschitz-continuously differentiable nonconvex function defined on a suitable compact set $\mathcal{C} \subset \mathbb{R}^{n}$ containing $\mathcal{D}$. Global optimization arises in many application areas including predicting the structures of proteins, managing financial porfolios, modelling chemical processes as well as managing environmental systems, to name but a few (see Floudas and Pardalos, 1999, for more application areas). While global optimization is a challenging problem (Kreinovich and Kearfott, 2005), a variety of deterministic and stochastic solution methods have been suggested (Androulakis, Maranas, and Floudas, 1995; Horst and Pardalos, 1995; Pardalos and Romeijn, 2002) and this remains a very active area of research (see Neumaier, 2004, and extensive references therein). Most of these approaches fall into one of three categories: exact or complete methods (mostly based on branch and bound, see Neumaier, 2004), heuristic or incomplete methods (mostly based on stochastic search, see Spall, 2003) and surrogate based methods (based on replacing the objective function by a cheap approximation, see Jones, 2001). Our approach to solving the global optimization problem (1.1) falls into the first category, it is a deterministic branch and bound algorithm drawing on established ideas from both the local and global optimization communities. To the best of our knowledge, it differs from other branch and bound algorithms in that it employs nonconvex underestimators of the function to obtain the required lower bounds.

The outline of the paper is as follows. In $\S 2$ we describe in detail our proposed branch and bound algorithm for solving the global optimization (1.1). We then go on to prove that under suitable assumptions the proposed algorithm converges to the global minimum of (1.1) in $\S 3$. $\S 4$ describes how we can efficiently calculate the necessary lower bounds required by our branch and bound algorithm while $\S 5$ outlines a faster heuristic version of our algorithm. Then in $\S 6$ we apply our proposed algorithm to radial basis functions and provide numerical examples to establish performance of the algorithm in this setting. Finally, we draw conclusions in $\S 7$.

\section{$1.1 \quad$ Notation}

Let $g(x):=\nabla_{x} f(x)$ and $H(x):=\nabla_{x x} f(x)$ be the gradient and Hessian of $f(x)$, and $\|\cdot\|$ denote the $\ell_{2}$-norm. Following convention, let $C^{k}$ denote the space of $k$-continuously differentiable functions. Since $\mathcal{C}$ is compact and $f \in C^{2}(\mathcal{C})$, there are constants $L>0$ and $L_{g}>0$ for which

$$
\|g(x)\| \leq L \text { and }\|H(x)\| \leq L_{g}
$$

for all $x \in \mathcal{C}$. It follows that $L$ is an $\ell_{2}$-norm Lipschitz constant for $f(x)$, and thus

$$
|f(x)-f(y)| \leq L\|x-y\|
$$

for all $x, y \in \mathcal{C}$, and that $L_{g}$ is a gradient Lipschitz constant for $f(x)$ over $\mathcal{C}$. Furthermore let $L_{H}$ be a Hessian Lipschitz constant of $f$ over $\mathcal{C}$. Note that, within $\mathcal{C}$, these are all global Lipschitz constants. 
Now let $\mathcal{B} \subset \mathcal{C}$ denote the $n$-dimensional closed ball of radius $r(\mathcal{B})>0$ centred at some $x_{\mathcal{B}} \in \mathcal{B}$, i.e.,

$$
\mathcal{B}=\left\{x \in \mathbb{R}^{n}:\left\|x-x_{\mathcal{B}}\right\| \leq r(\mathcal{B})\right\} .
$$

Furthermore, let $L_{H}(\mathcal{B})$ denote a local Hessian Lipschitz constant for $f(x)$ over the ball $\mathcal{B}$. Unlike the global Lipschitz constants, we need to be able to calculate a numerical approximation to the local Hessian Lipschitz constant for our proposed branch and bound algorithm. We discuss how this is done in detail in an application to radial basis functions in $§ 6.2$.

\section{Description of the Algorithm}

In an attempt to solve the global optimization problem (1.1) we develop an extension of the canonical branch-and-bound algorithm (see e.g. Horst, 1986) with bounds inspired by the trust region subproblem (see Chapter 7 of Conn, Gould, and Toint, 2000). Our branching comprises a systematic covering and refinement of $\mathcal{D}$ by balls $\mathcal{B}$, while our bounding requires we compute both lower and upper bounds on the minimum of $f$ over each $\mathcal{B}$. We will return to the branching when we define our algorithm.

To find a lower bound for the minimum of $f$ over $\mathcal{B}$, we deduce from Taylor's theorem that for all $x, x_{\mathcal{B}} \in \mathcal{B}$

$$
\begin{aligned}
f(x)= & f\left(x_{\mathcal{B}}\right)+\left(x-x_{\mathcal{B}}\right)^{T} g\left(x_{\mathcal{B}}\right)+\frac{1}{2}\left(x-x_{\mathcal{B}}\right)^{T} H\left(x_{\mathcal{B}}\right)\left(x-x_{\mathcal{B}}\right) \\
& +\int_{0}^{1}(1-\tau)\left(x-x_{\mathcal{B}}\right)^{T}\left[H\left(x_{\mathcal{B}}+\tau\left(x-x_{\mathcal{B}}\right)\right)-H\left(x_{\mathcal{B}}\right)\right]\left(x-x_{\mathcal{B}}\right) d \tau .
\end{aligned}
$$

Taking the absolute value of the integral and invoking the Hessian Lipschitz continuity, we find that for all $x \in \mathcal{B}$

$$
\begin{aligned}
& \left|\int_{0}^{1}(1-\tau)\left(x-x_{\mathcal{B}}\right)^{T}\left[H\left(x_{\mathcal{B}}+\tau\left(x-x_{\mathcal{B}}\right)\right)-H\left(x_{\mathcal{B}}\right)\right]\left(x-x_{\mathcal{B}}\right) d \tau\right| \\
& \leq \int_{0}^{1}(1-\tau)\left\|H\left(x_{\mathcal{B}}+\tau\left(x-x_{\mathcal{B}}\right)\right)-H\left(x_{\mathcal{B}}\right)\right\|\left\|x-x_{\mathcal{B}}\right\|^{2} d \tau \\
& \leq \int_{0}^{1}(1-\tau) L_{H}(\mathcal{B})\left\|\tau\left(x-x_{\mathcal{B}}\right)\right\|\left\|x-x_{\mathcal{B}}\right\|^{2} d \tau \\
& =\int_{0}^{1}(1-\tau) \tau d \tau L_{H}(\mathcal{B})\left\|x-x_{\mathcal{B}}\right\|^{3}=\frac{L_{H}(\mathcal{B})}{6}\left\|x-x_{\mathcal{B}}\right\|^{3} .
\end{aligned}
$$

Thus if we consider the lower cubic bounding function $m_{\mathcal{B}}^{-}: \mathcal{B} \subset \mathbb{R}^{n} \rightarrow \mathbb{R}$ (as in Nesterov and Polyak, 2006)

$$
m_{\mathcal{B}}^{-}(x)=f\left(x_{\mathcal{B}}\right)+\left(x-x_{\mathcal{B}}\right)^{T} g\left(x_{\mathcal{B}}\right)+\frac{1}{2}\left(x-x_{\mathcal{B}}\right)^{T} H\left(x_{\mathcal{B}}\right)\left(x-x_{\mathcal{B}}\right)-\frac{L_{H}(\mathcal{B})}{6}\left\|x-x_{\mathcal{B}}\right\|^{3},
$$

we have that

$$
m_{\mathcal{B}}^{-}(x) \leq f(x)
$$

provides a non-convex under-estimator for all $x \in \mathcal{B}$. We can therefore use

$$
\alpha(\mathcal{B})=\min _{x \in \mathcal{B}} m_{\mathcal{B}}^{-}(x)
$$


as a lower bound for the global minimum of $f(x)$ over $\mathcal{B}$. It is important to note that $\alpha(\mathcal{B})$ can be calculated efficiently and we discuss how this is done in $\S 4$.

It is also possible (when $g\left(x_{\mathcal{B}}\right) \neq 0$ ) to use a lower bound based on the gradient Lipschitz constant

$$
f\left(x_{\mathcal{B}}\right)+\left(x-x_{\mathcal{B}}\right)^{T} g\left(x_{\mathcal{B}}\right)-\frac{L_{g}(\mathcal{B})}{2}\left\|x-x_{\mathcal{B}}\right\|^{2}
$$

although numerical results suggest the cubic lower bounding function detailed above almost always provides a tighter underestimator. Nevertheless, as it is faster to compute it may be more efficient to use this quadratic lower bound in the early stages of the branch and bound algorithm when the cubic lower bound is rather crude.

To find an upper bound for the minimum of $f$ over $\mathcal{B}$, we simply evaluate $f$ at a feasible point $x_{\mathcal{B}}^{+}$in $\mathcal{B}$. We discuss how $x_{\mathcal{B}}^{+}$is calculated in $\S 2.1$. We therefore let

$$
\beta(\mathcal{B})=f\left(x_{\mathcal{B}}^{+}\right)
$$

be the upper bound for the global minimum of $f(x)$ over $\mathcal{B}$.

The idea behind the algorithm is to recursively partition an initial ball covering the domain $\mathcal{D}$ into sub-balls until we find a ball (or balls) of sufficiently small size containing the global minimiser of $f(x)$ over $\mathcal{D}$. Since we are able to obtain bounds on the minimum of $f(x)$ over any ball in $\mathcal{D}$, we can use them to discard balls which cannot contain the global minimum, i.e. balls whose lower bound is greater than the smallest upper bound. The complete branch and bound algorithm then proceeds as follows:

\section{Algorithm 2.1. Branch and Bound Algorithm for Hessian Lipschitz Optimization}

0. Initialisation:

(a) Set $k=0$.

(b) Let $\mathcal{B}_{0}$ be a ball with centre $x_{\mathcal{B}} \in \mathcal{D}$ of sufficiently large radius to cover $\mathcal{D}$.

(c) Let $\mathcal{L}_{0}=\left\{\mathcal{B}_{0}\right\}$ be the initial list of balls.

(d) Let $U_{0}=\beta\left(\mathcal{B}_{0}\right)$ be the initial upper bound for $\min _{x \in \mathcal{D}} f(x)$.

(e) Let $L_{0}=\alpha\left(\mathcal{B}_{0}\right)$ be the initial lower bound for $\min _{x \in \mathcal{D}} f(x)$.

1. While $U_{k}-L_{k}>\varepsilon$, repeat the following procedure:

(a) Remove from $\mathcal{L}_{k}$ balls $\mathcal{B} \in \mathcal{L}_{k}$ such that $\alpha(\mathcal{B})>U_{k}$.

(b) Choose $\mathcal{B} \in \mathcal{L}_{k}$ such that $\alpha(\mathcal{B})=L_{k}$.

(c) Split $\mathcal{B}$ into $3^{n}$ overlapping sub-balls $\mathcal{B}_{1}, \ldots, \mathcal{B}_{3^{n}}$ according to our splitting rule (see §2.2) and discard any sub-balls which lie entirely outside of $\mathcal{D}$. Let $\mathcal{R}_{k}$ denote the list of remaining sub-balls and let $\mathcal{L}_{k+1}:=\left(\mathcal{L}_{k} \backslash\{\mathcal{B}\}\right) \cup \mathcal{R}_{k}$.

(d) Set $U_{k+1}:=\min _{\mathcal{B} \in \mathcal{L}_{k+1}} \beta(\mathcal{B})$.

(e) Set $L_{k+1}:=\min _{\mathcal{B} \in \mathcal{L}_{k+1}} \alpha(\mathcal{B})$.

(f) Set $k=k+1$.

2. Return $U_{k}$ as the estimate of the global minimum of $f(x)$ over $\mathcal{D}$.

Note that infeasible balls, i.e. balls which lie entirely outside of $\mathcal{D}$, are discarded by the branch and bound algorithm (see step 1c) and that the initial ball $\mathcal{B}_{0}$ contains $\mathcal{D}$. 


\subsection{Discarding Balls and Feasible Points}

Algorithm 2.1 discards balls $\mathcal{B}$ which lie entirely outside of $\mathcal{D}$. As $\mathcal{D}$ is a convex set this is easy to check since then the convex programming problem

$$
\begin{aligned}
& \min _{x \in \mathbb{R}^{n}}\left\|x-x_{\mathcal{B}}\right\|^{2} \\
& \text { s.t. } x \in \mathcal{D}
\end{aligned}
$$

provides a feasible minimiser $x_{\mathcal{B}}^{+}$if the minimum is smaller than $r(\mathcal{B})^{2}$. Moreover, if the minimum of the convex program is larger than $r(\mathcal{B})^{2}$, we know that the ball $\mathcal{B}$ lies entirely outside of $\mathcal{D}$ and can be discarded. The convex programming problem can be efficiently solved using standard convex optimization techniques (see Boyd and Vandenberghe, 2004).

\subsection{Splitting Rule}

We split a ball $\mathcal{B} \subset \mathbb{R}^{n}$ in step 1c of Algorithm 2.1 as follows. Let $\mathcal{B}$ have centre $x_{\mathcal{B}}$ and radius $r(\mathcal{B})$. Split $\mathcal{B}$ into $3^{n}$ sub-balls of radius $r(\mathcal{B}) / 2$ centred at the vertices of a hypercubic tessellation around $x_{\mathcal{B}}$ of edge length $r(\mathcal{B}) / \sqrt{n}$. Formally, construct $3^{n}$ sub-balls $\mathcal{B}_{1}, \ldots, \mathcal{B}_{3^{n}}$ all of radius $r(\mathcal{B}) / 2$ centred at

$$
x_{\mathcal{B}_{i}}=x_{\mathcal{B}}+\rho_{i}^{n}\left(\frac{-r(\mathcal{B})}{\sqrt{n}}, 0, \frac{r(\mathcal{B})}{\sqrt{n}}\right)
$$

for $i=1, \ldots, 3^{n}$. Here $\rho_{i}^{n}\left(s_{1}, s_{2}, s_{3}\right)$ is a vector in $\mathbb{R}^{n}$ whose elements are the $i$-th permutation of $s_{1}, s_{2}, s_{3}$ taken $n$ at a time with repetition. We illustrate this for the case $n=2$ in Figure 1 . Note that the choice of centres and radii of the sub-balls ensures that they cover the original ball $\mathcal{B}$. Furthermore, this means that at any iteration of Algorithm 2.1 we always have a covering of closed balls of the convex set $\mathcal{D}$.

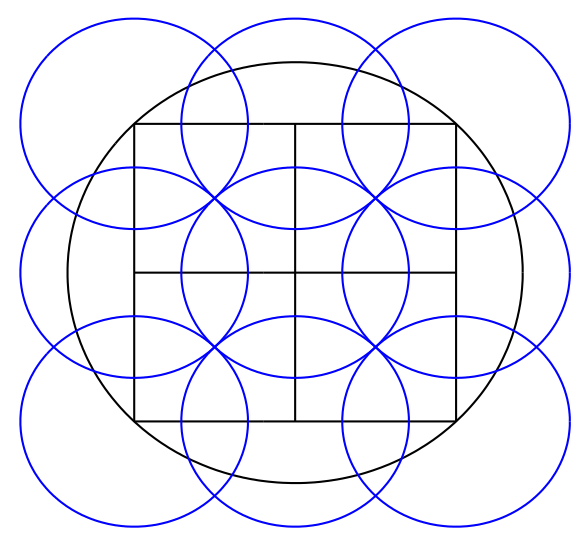

Figure 1: An illustration of our splitting rule in two dimensions. The black circle is split into nine blue circles of half radius centred at the vertices of the square tessellation. 


\section{Proof of Convergence}

In this section we will prove that, under suitable assumptions, Algorithm 2.1 converges in a finite number of iterations to within a tolerance $\varepsilon>0$ of the global minimum of $f(x)$. Our proof is based on the convergence proof of the canonical box-based bound constrained branch and bound algorithm in Balakrishnan, Boyd, and Balemi (1991). First we state and prove a pair of Lemmata before giving the main convergence theorem.

Lemma 3.1. The bounds $\alpha$ and $\beta$ given above in (2.3) and (2.4) respectively satisfy

$(C 1) \alpha(\mathcal{B}) \leq \min _{x \in \mathcal{B}} f(x) \leq \beta(\mathcal{B}) \quad \forall \mathcal{B} \subset \mathbb{R}^{n}$

(C2) $\forall \varepsilon>0 \exists \delta>0$ s.t. $\forall \mathcal{B} \subset \mathbb{R}^{n}, r(\mathcal{B}) \leq \delta \Longrightarrow \beta(\mathcal{B})-\alpha(\mathcal{B}) \leq \varepsilon$

Proof: $\quad(\mathrm{C} 1)$ Recall that $\alpha(\mathcal{B})=\min _{x \in \mathcal{B}} m_{\mathcal{B}}^{-}(x)$ and $\beta(\mathcal{B})=f\left(x_{\mathcal{B}}^{+}\right)$. From $(2.2)$ we have that

$$
\min _{x \in \mathcal{B}} m_{\mathcal{B}}^{-}(x) \leq \min _{x \in \mathcal{B}} f(x)
$$

and clearly

$$
\min _{x \in \mathcal{B}} f(x) \leq f\left(x_{\mathcal{B}}^{+}\right) .
$$

Thus we see that the bounds satisfy condition (C1).

(C2) Let $\varepsilon>0$ be arbitrary. For clarity of exposition define for all $\mathcal{B} \subset \mathbb{R}^{n}$,

$$
x_{\mathcal{B}}^{-}:=\arg \min _{x \in \mathcal{B}} m_{\mathcal{B}}^{-}(x) \text {. }
$$

Note that $x_{\mathcal{B}}^{-}$may not be unique but this does not matter. Then $r(\mathcal{B}) \leq \delta$ means that

$$
\left\|x_{\mathcal{B}}^{-}-x_{\mathcal{B}}\right\| \leq \delta
$$

Consider

$$
\begin{aligned}
\beta(\mathcal{B})-\alpha(\mathcal{B})= & \left|f\left(x_{\mathcal{B}}^{+}\right)-m_{\mathcal{B}}^{-}\left(x_{\mathcal{B}}^{-}\right)\right| \\
\leq & \left|f\left(x_{\mathcal{B}}^{+}\right)-f\left(x_{\mathcal{B}}\right)\right|+\left\|x_{\mathcal{B}}^{-}-x_{\mathcal{B}}\right\|\left\|g\left(x_{\mathcal{B}}\right)\right\| \\
& +\frac{1}{2}\left\|x_{\mathcal{B}}^{-}-x_{\mathcal{B}}\right\|^{2}\left\|H\left(x_{\mathcal{B}}\right)\right\|+\frac{L_{H}}{6}\left\|x_{\mathcal{B}}^{-}-x_{\mathcal{B}}\right\|^{3} \\
\leq & L\left\|x_{\mathcal{B}}^{+}-x_{\mathcal{B}}\right\|+\left\|x_{\mathcal{B}}^{-}-x_{\mathcal{B}}\right\|\left\|g\left(x_{\mathcal{B}}\right)\right\| \\
& +\frac{1}{2}\left\|x_{\mathcal{B}}^{-}-x_{\mathcal{B}}\right\|^{2}\left\|H\left(x_{\mathcal{B}}\right)\right\|+\frac{L_{H}}{6}\left\|x_{\mathcal{B}}^{-}-x_{\mathcal{B}}\right\|^{3} \\
\leq & L \delta+\left\|g\left(x_{\mathcal{B}}\right)\right\| \delta+\frac{1}{2}\left\|H\left(x_{\mathcal{B}}\right)\right\| \delta^{2}+\frac{L_{H}}{6} \delta^{3} \\
\leq & 2 L \delta+\frac{L_{g}}{2} \delta^{2}+\frac{L_{H}}{6} \delta^{3}
\end{aligned}
$$

where the first inequality follows directly from the triangle and Cauchy-Schwarz inequalities, the second from (1.3), the third from (3.2) and the fourth from (1.2). It therefore suffices to choose $\delta$ sufficiently small such that

$$
2 L \delta+\frac{L_{g}}{2} \delta^{2}+\frac{L_{H}}{6} \delta^{3} \leq \varepsilon
$$


so we simply need to pick $\delta \leq \min \left\{\varepsilon / 6 L, \sqrt{2 \varepsilon / 3 L_{g}}, \sqrt[3]{2 \varepsilon / L_{H}}\right\}$, i.e. such that each of the three terms on the left of the bounding equation for $\varepsilon$ is less than or equal to $\varepsilon / 3$.

Our second Lemma shows that Algorithm 2.1 eventually creates a ball of arbitrarily small radius.

Lemma 3.2. For $k \in \mathbb{N}$

$$
\min _{\mathcal{B} \in \mathcal{L}_{k}} r(\mathcal{B}) \leq \frac{r\left(\mathcal{B}_{0}\right)}{2^{\left\lceil\left(\log _{3} n k\right) / 2\right\rceil}}
$$

and thus for any $\delta>0$ there exists $K \in \mathbb{N}$ such that

$$
\min _{\mathcal{B} \in \mathcal{L}_{K}} r(\mathcal{B}) \leq \delta .
$$

Proof: Firstly recall that our splitting rule splits each ball into $3^{n}$ sub-balls. We start at iteration $k=0$ with our initial covering ball $\mathcal{B}_{0}$ of radius $r\left(\mathcal{B}_{0}\right)$. We split $\mathcal{B}_{0}$ into $3^{n}$ sub-balls of radius $r\left(\mathcal{B}_{0}\right) / 2$ at iteration $k=1$. Assuming a worst-case scenario, each of these $3^{n}$ sub-balls has to be split into $3^{n}$ subsub-balls of radius $r\left(\mathcal{B}_{0}\right) / 4$ before we can consider any of the subsubballs for splitting. Following this argument through inductively, we deduce that for $k \in \mathbb{N}$ it takes at most

$$
k=\sum_{j=1}^{m}\left(3^{n}\right)^{j-1}
$$

iterations to reduce the radius of the smallest ball in the covering to less than or equal to

$$
\frac{r\left(\mathcal{B}_{0}\right)}{2^{m}} \text {. }
$$

We can bound (3.3) by

$$
k \leq m\left(3^{n}\right)^{m-1} \leq\left(3^{n}\right)^{2 m}
$$

which when combined with (3.4) gives the required bound. The second part of the Lemma then follows trivially.

Theorem 3.3. Algorithm 2.1 converges in a finite number of iterations to within a tolerance $\varepsilon>0$ of the global minimum of $f(x)$ over $\mathcal{D}$. Formally, for any $\varepsilon>0$ there exists $M_{\varepsilon} \in \mathbb{N}$ such that

$$
U_{M_{\varepsilon}}-L_{M_{\varepsilon}} \leq \varepsilon
$$

and $U_{M_{\varepsilon}}$ is within a tolerance $\varepsilon$ of the global minimum of $f(x)$ over $\mathcal{D}$.

Proof: Let $\mathcal{B}_{k}:=\arg \min _{\mathcal{B} \in \mathcal{L}_{k}} r(\mathcal{B})$ and let $\mathcal{A}_{k} \in \mathcal{L}_{M_{k}}$ be the ball which for some $M_{k}<k$ we split to obtain $\mathcal{B}_{k}$. Note that $\mathcal{B}_{k}$ may not be unique but this does not matter. Let $\varepsilon>0$ be arbitrary. Then there exists $\delta>0$ such that for any $\mathcal{B} \subset \mathbb{R}^{n}$

$$
r(\mathcal{B}) \leq 2 \delta \Longrightarrow \beta(\mathcal{B})-\alpha(\mathcal{B}) \leq \varepsilon
$$

by condition (C2) of Lemma 3.1. Choose $K \in \mathbb{N}$ sufficiently large such that

$$
r\left(\mathcal{B}_{K}\right) \leq \delta
$$


which is possible by Lemma 3.2. Then $\mathcal{A}_{K}$ must have $r\left(\mathcal{A}_{K}\right) \leq 2 \delta$ as we split it to obtain $\mathcal{B}_{k}$ and thus from (3.5) we have that

$$
\beta\left(\mathcal{A}_{K}\right)-\alpha\left(\mathcal{A}_{K}\right) \leq \varepsilon .
$$

Now, as $\mathcal{A}_{K}$ was split at iteration $M_{K}$, it must have satisfied $\alpha\left(\mathcal{A}_{K}\right)=L_{M_{K}}$. Hence we get that

$$
U_{M_{K}}-L_{M_{K}} \leq \beta\left(\mathcal{A}_{K}\right)-L_{M_{K}} \leq \varepsilon
$$

since $U_{M_{K}} \leq \beta\left(\mathcal{A}_{K}\right)$ by definition and using (3.6). We therefore have an upper bound $M_{K}$ on the number of branch and bound iterations.

It remains to show that $U_{M_{K}}$ is within a tolerance $\varepsilon$ of the global minimum of $f(x)$ over $\mathcal{D}$. Assume condition (C1) of Lemma 3.1 holds and suppose that the global minimum $l^{*}$ of $f(x)$ over $\mathcal{D}$ is attained at $x^{*} \in \mathcal{D}$. First, we show that $x^{*}$ is contained in a ball in $\mathcal{L}_{M_{K}}$. To see this, observe that for all $k \in \mathbb{N}, \mathcal{L}_{k}$ is a partition of the bounding ball $\mathcal{B}_{0}$ with balls which cannot possibly contain $x^{*}$ removed, that is to say balls $\mathcal{B}$ which have lower bound

$$
\alpha(\mathcal{B})>U_{k}
$$

i.e. $\alpha(\mathcal{B})>f\left(x_{\mathcal{B}}^{+}\right)$for a feasible point $x_{\mathcal{B}}^{+} \in \mathcal{D}$. As $x^{*}$ is contained in a ball in $\mathcal{L}_{M_{K}}$ it follows that $L_{M_{K}} \leq l^{*}$ and thus

$$
U_{M_{K}}-l^{*} \leq U_{M_{K}}-L_{M_{K}} \leq \varepsilon
$$

by (3.7).

\section{Computing the Lower Bound}

We have mentioned earlier in $\S 2$ that we use lower bounds based on globally minimising the cubic bounding function $m_{\mathcal{B}}^{-}(x)$ over balls $\mathcal{B} \subset \mathbb{R}^{n}$, in a similar vein to Nesterov and Polyak (2006). In this subsection we will show how we can efficiently globally minimise $m_{\mathcal{B}}^{-}(x)$ over any closed ball $\mathcal{B}$ centred at $x_{\mathcal{B}}$, i.e.

$$
\begin{aligned}
& \text { minimise } m_{\mathcal{B}}^{-}(x)= f\left(x_{\mathcal{B}}\right)+\left(x-x_{\mathcal{B}}\right)^{T} g\left(x_{\mathcal{B}}\right)+\frac{1}{2}\left(x-x_{\mathcal{B}}\right)^{T} H\left(x_{\mathcal{B}}\right)\left(x-x_{\mathcal{B}}\right) \\
&-\frac{L_{H}(\mathcal{B})}{6}\left\|x-x_{\mathcal{B}}\right\|^{3} \\
& \text { subject to }\left\|x-x_{\mathcal{B}}\right\| \leq \Delta
\end{aligned}
$$

for some $\Delta>0$. For clarity of exposition, we rewrite the above minimisation problem in the equivalent form

$$
\begin{aligned}
& \text { minimise } m^{-}(x):=f+x^{T} g+\frac{1}{2} x^{T} H x-\frac{\sigma}{3}\|x\|^{3} \\
& \text { subject to }\|x\| \leq \Delta
\end{aligned}
$$

where $\sigma:=L_{H}(\mathcal{B}) / 2$, we have shifted $x$ by $x_{\mathcal{B}}$, and we have dropped the explicit dependence on $\mathcal{B}$ from the notation. It is clear that the global minimum of the above problem will occur either on the boundary or in the interior of the $\Delta$-ball. We solve for these two cases in turn, starting with the case where the minimum lies on the boundary. 


\subsection{Finding a minimiser on the $\Delta$-ball boundary}

For the bounding function $\mathrm{m}^{-}(x)$ we have the following global optimality result (cf. Theorem 7.2.1 in Conn et al., 2000 and Theorem 3.1 in Cartis, Gould, and Toint, 2009).

Theorem 4.1. Any $x^{*}$ is a global minimiser of $m^{-}(x)$ over $\mathbb{R}^{n}$ subject to $\|x\|=\Delta$ if and only if it satisfies the system of equations

$$
\left(H+\left(\lambda^{*}-\sigma \Delta\right) I\right) x^{*}=-g
$$

where $H+\left(\lambda^{*}-\sigma \Delta\right) I$ is positive semidefinite for some Lagrange multiplier $\lambda^{*}$ and $\left\|x^{*}\right\|=\Delta$. If $H+\left(\lambda^{*}-\sigma \Delta\right) I$ is positive definite, $x^{*}$ is unique.

Proof: First we rewrite the constraint $\|x\|=\Delta$ as $\frac{1}{2}\|x\|^{2}-\frac{1}{2} \Delta^{2}=0$. Now, let $x^{*}$ be a global minimiser of $m^{-}(x)$ over $\mathbb{R}^{n}$ subject to the constraint. We have from the first order necessary optimality conditions (see Section 3.2.2 of Conn et al., 2000) that $x^{*}$ satisfies

$$
\left(H+\left(\lambda^{*}-\sigma\left\|x^{*}\right\|\right) I\right) x^{*}=-g .
$$

where $\lambda^{*}$ is the corresponding Lagrange multiplier. We have by assumption that $\left\|x^{*}\right\|=\Delta$ and substituting this into (4.2) gives the required system (4.1). Now, suppose $u^{*}$ is a feasible point, i.e. that $\left\|u^{*}\right\|=\Delta$. We have that

$$
\begin{aligned}
m^{-}\left(u^{*}\right)-m^{-}\left(x^{*}\right) & =g^{T}\left(u^{*}-x^{*}\right)+\frac{1}{2}\left(u^{*}\right)^{T} H u^{*}-\frac{1}{2}\left(x^{*}\right)^{T} H x^{*}+\frac{\sigma}{3}\left(\left\|u^{*}\right\|^{3}-\left\|x^{*}\right\|^{3}\right) \\
& =g^{T}\left(u^{*}-x^{*}\right)+\frac{1}{2}\left(u^{*}\right)^{T} H u^{*}-\frac{1}{2}\left(x^{*}\right)^{T} H x^{*}
\end{aligned}
$$

where the last equality follows from the fact that $\left\|x^{*}\right\|=\left\|u^{*}\right\|=\Delta$. But (4.2) gives that

$$
g^{T}\left(u^{*}-x^{*}\right)=\left(x^{*}-u^{*}\right)^{T} H x^{*}+\left(\lambda^{*}-\sigma \Delta\right)\left(x^{*}-u^{*}\right)^{T} x^{*} .
$$

Also, the fact that $\left\|x^{*}\right\|=\left\|u^{*}\right\|=\Delta$ implies that

$$
\left(x^{*}-u^{*}\right)^{T} x^{*}=\frac{1}{2}\left(x^{*}\right)^{T} x^{*}+\frac{1}{2}\left(u^{*}\right)^{T} u^{*}-\left(u^{*}\right)^{T} x^{*}=\frac{1}{2}\left(u^{*}-x^{*}\right)^{T}\left(u^{*}-x^{*}\right) .
$$

Combining (4.3) with (4.4) and (4.5), we find that

$$
\begin{aligned}
m^{-}\left(u^{*}\right)-m^{-}\left(x^{*}\right)= & \frac{1}{2}\left(\lambda^{*}-\sigma \Delta\right)\left(u^{*}-x^{*}\right)^{T}\left(u^{*}-x^{*}\right)+\frac{1}{2}\left(u^{*}\right)^{T} H u^{*} \\
& -\frac{1}{2}\left(x^{*}\right)^{T} H x^{*}+\left(x^{*}\right)^{T} H x^{*}-\left(u^{*}\right)^{T} H x^{*} \\
= & \frac{1}{2}\left(u^{*}-x^{*}\right)^{T}\left(H+\left(\lambda^{*}-\sigma \Delta\right) I\right)\left(u^{*}-x^{*}\right) .
\end{aligned}
$$

We also have from the second order necessary optimality conditions (see Section 3.2.2 of Conn et al., 2000) that

$$
H+\left(\lambda^{*}-\sigma\left\|x^{*}\right\|\right) I-\frac{\sigma}{\left\|x^{*}\right\|} x^{*}\left(x^{*}\right)^{T}
$$


is positive semidefinite on the null-space of the constraint gradient $x^{*}$, i.e. that

$$
v^{T}\left(H+\left(\lambda^{*}-\sigma \Delta\right) I-\frac{\sigma}{\Delta} x^{*}\left(x^{*}\right)^{T}\right) v \geq 0
$$

for all $v$ for which $v^{T} x^{*}=0$, where we have used the fact that $\left\|x^{*}\right\|=\Delta$. In this case it immediately follows from (4.7) that

$$
v^{T}\left(H+\left(\lambda^{*}-\sigma \Delta\right) I\right) v \geq 0
$$

for all $v$ for which $v^{T} x^{*}=0$. It thus remains to consider vectors $v$ for which $v^{T} x^{*} \neq 0$. Since $v$ and $x^{*}$ are not orthogonal, the line $x^{*}+\alpha v$ intersects the constraint $\|x\|=\Delta$ at two points, $x^{*}$ and $u^{*}$. Let $v^{*}=u^{*}-x^{*}$ and note that $v^{*}$ is parallel to $v$. As $x^{*}$ is a global minimiser we have that $m^{-}\left(u^{*}\right) \geq m^{-}\left(x^{*}\right)$ and thus we have from (4.6) that

$$
\begin{aligned}
0 \leq m^{-}\left(u^{*}\right)-m^{-}\left(x^{*}\right) & =\frac{1}{2}\left(u^{*}-x^{*}\right)^{T}\left(H+\left(\lambda^{*}-\sigma \Delta\right) I\right)\left(u^{*}-x^{*}\right) \\
& =\frac{1}{2}\left(v^{*}\right)^{T}\left(H+\left(\lambda^{*}-\sigma \Delta\right) I\right) v^{*}
\end{aligned}
$$

from which we deduce that

$$
v^{T}\left(H+\left(\lambda^{*}-\sigma \Delta\right) I\right) v \geq 0
$$

for all $v$ for which $v^{T} x^{*} \neq 0$. In summary, we have shown that

$$
v^{T}\left(H+\left(\lambda^{*}-\sigma \Delta\right) I\right) v \geq 0
$$

for any vector, which is the same as saying that $H+\left(\lambda^{*}-\sigma \Delta\right) I$ must be positive semidefinite. Conversely, if $H+\left(\lambda^{*}-\sigma \Delta\right) I$ is positive definite, $\frac{1}{2}\left(u^{*}-x^{*}\right)^{T}\left(H+\left(\lambda^{*}-\sigma \Delta\right) I\right)\left(u^{*}-x^{*}\right)>0$ for any $u^{*} \neq x^{*}$ and therefore (4.8) shows that $m^{-}\left(u^{*}\right)>m^{-}\left(x^{*}\right)$ whenever $u^{*}$ is feasible. Thus $x^{*}$ is the unique global minimiser.

The global minimiser can be efficiently found by applying a safeguarded version of Newton's method as detailed in Section 2.1 of Gould, Robinson, and Thorne (2010) to the scalar equation

$$
\begin{aligned}
\|x(\lambda)\| & =\Delta, \\
\text { where }(H+(\lambda-\sigma \Delta) I) x(\lambda) & =-g,
\end{aligned}
$$

and this is the approach we take (see Figure 2 below). Note that since $H$ is of low dimension it is more efficient to find the spectral decomposition $H=Q \Lambda Q^{T}$, and then to solve an equivalent problem to (4.9) in transformed variables $y=Q^{T} x$ for which the Hessian, $\Lambda$, is diagonal.

\subsection{Finding a minimiser in the $\Delta$-ball interior}

If, by contrast, the solution we seek lies in the interior of $\mathcal{B}$, we have the following result:

Theorem 4.2. The lower bounding function $m^{-}(x)$ can only have finite global minimisers if $H$ is positive semidefinite. In this case, any $x^{*}$ is a global minimiser of $m^{-}(x)$ over $\mathbb{R}^{n}$ if and only if it satisfies the system of equations

$$
\left(H+\omega^{*} I\right) x^{*}=-g
$$

where $\omega^{*}=-\sigma\left\|x^{*}\right\|$ and $H+\omega^{*} I$ is positive semidefinite. 


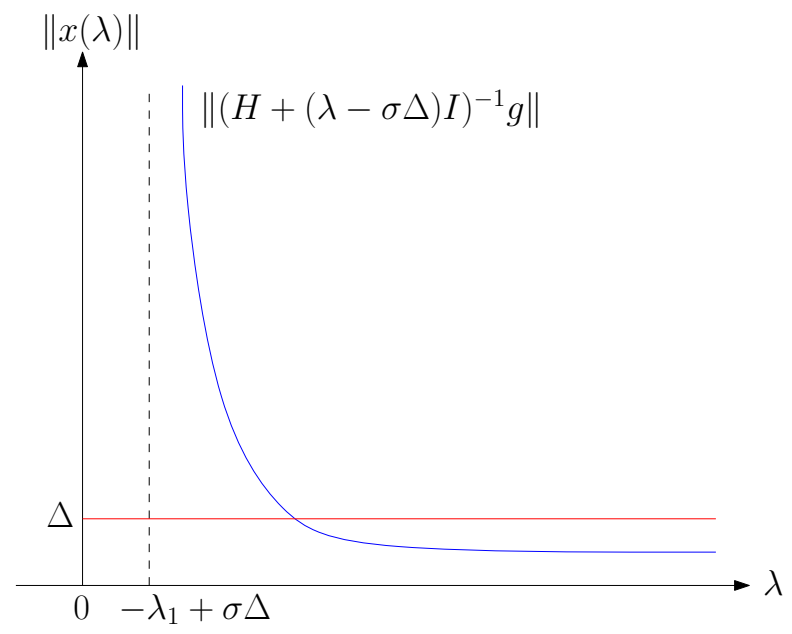

Figure 2: Solutions to the system (4.1) are the intersections of the two curves.

Proof: For the first part, suppose $H$ is not positive semidefinite and consider $x=\alpha u$ for any eigenvector $u$ of $H$ corresponding to a negative eigenvalue. Then clearly $m^{-}(x) \rightarrow-\infty$ as $\alpha \rightarrow \infty$ and so the function $m^{-}(x)$ is unbounded below. The second part of the proof is analogous to the proof of the first part of Theorem 3.1 in Cartis et al. (2009).

Note that in this case $\omega^{*}=-\sigma\left\|x^{*}\right\|$ and so there can only be a solution for $\omega \leq 0$. Assuming $H$ is positive semidefinite, let $\lambda_{1}$ denote the smallest eigenvalue of $H$ and note that if $\lambda_{1}=0$ there can only be a trivial solution to the system (4.10) when $x=0$ and $g=0$. When $\lambda_{1}>0$ there will be at most two possible solutions to the system (4.10) which, once again, can be found using Newton's method with suitable starting points (i.e. Algorithm 6.1 in Cartis et al., 2009). Numerical results suggest that in this case the solution closest to zero is always the best local minimiser in the $\Delta$-ball interior, and this is indeed the case as we show in Theorem 4.3 below. Figure 3 below illustrates this typical case when there are two possible solutions. (Note that there may be no solutions and an example of this is the case where the straight line lies under the curve in Figure 3.) We have the following theorem (based on Theorem 3 from

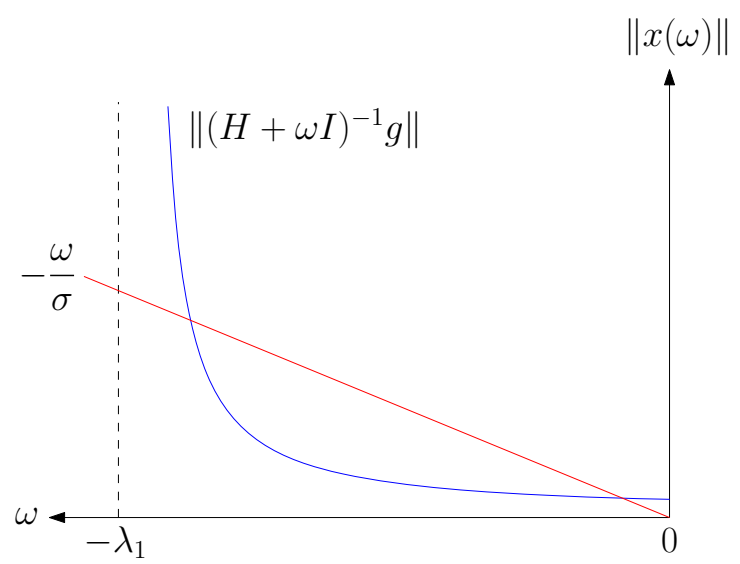

Figure 3: Solutions to the system (4.10) for $m^{-}(x)$ are the intersections of the two curves.

Griewank, 1981) which shows that whenever $m^{-}(x)$ has a finite global minimiser over $\mathbb{R}^{n}$, the global minimiser is unique. 
Theorem 4.3. For any pair of potential finite global minimisers $u^{*}, v^{*}$ of the lower bounding function $m^{-}(x)$ over $\mathbb{R}^{n}$ given by Theorem 4.2 with $\left\|u^{*}\right\| \leq\left\|v^{*}\right\|$, the corresponding lower bounding function values satisfy

$$
m^{-}\left(v^{*}\right)-m^{-}\left(u^{*}\right) \geq \frac{\sigma}{6}\left(\left\|v^{*}\right\|-\left\|u^{*}\right\|\right)^{3} \geq 0 .
$$

In particular this means that when there are two possible solutions to the system (4.10) (as in Figure 3), the solution $x^{*}$ with larger norm $\left\|x^{*}\right\|$ is the global minimiser of $m^{-}(x)$ over $\mathbb{R}^{n}$.

Proof: First of all consider a general potential finite global minimiser $x^{*}$ of $m^{-}(x)$. As it is a potential finite global minimiser it must satisfy (4.10). Multiplying (4.10) on the left by $\left(x^{*}\right)^{T}$ and dividing by two gives

$$
\frac{1}{2}\left(x^{*}\right)^{T} H x^{*}=-\frac{1}{2} g^{T} x^{*}-\frac{1}{2} \omega^{*}\left(x^{*}\right)^{T} x^{*}
$$

where $\omega^{*}=-\sigma\left\|x^{*}\right\|$. From the definition of $m^{-}(x)$ we have that

$$
\begin{aligned}
m^{-}\left(x^{*}\right) & =f+g^{T} x^{*}+\frac{1}{2}\left(x^{*}\right)^{T} H x^{*}-\frac{\sigma}{3}\left\|x^{*}\right\|^{3} \\
& =f+g^{T} x^{*}-\frac{1}{2} g^{T} x^{*}-\frac{1}{2} \omega^{*}\left(x^{*}\right)^{T} x^{*}-\frac{\sigma}{3}\left\|x^{*}\right\|^{3} \quad \text { by }(4.11) \\
& =f+\frac{1}{2} g^{T} x^{*}+\frac{\sigma}{2}\left\|x^{*}\right\|\left\|x^{*}\right\|^{2}-\frac{\sigma}{3}\left\|x^{*}\right\|^{3} \\
& =f+\frac{1}{2} g^{T} x^{*}+\frac{\sigma}{6}\left\|x^{*}\right\|^{3}
\end{aligned}
$$

where the third equality follows from the fact that $\omega^{*}=-\sigma\left\|x^{*}\right\|$ and $\left(x^{*}\right)^{T} x^{*}=\left\|x^{*}\right\|^{2}$. Now, assume $u^{*}, v^{*}$ are potential finite global minimisers of $m^{-}(x)$. We have from Theorem 4.2 that

$$
\left(H+\alpha^{*} I\right) u^{*}=-g=\left(H+\beta^{*} I\right) v^{*}
$$

where

$$
\alpha^{*}=-\sigma\left\|u^{*}\right\| \geq-\sigma\left\|v^{*}\right\|=\beta^{*} .
$$

Multiplying (4.13) by $\left(v^{*}\right)^{T}$ and $\left(u^{*}\right)^{T}$ we obtain

$$
\begin{aligned}
& -g^{T} v^{*}=\left(v^{*}\right)^{T} H u^{*}+\alpha^{*}\left(v^{*}\right)^{T} u^{*} \\
& -g^{T} u^{*}=\left(u^{*}\right)^{T} H v^{*}+\beta^{*}\left(u^{*}\right)^{T} v^{*}
\end{aligned}
$$

so that (since $H$ is symmetric)

$$
g^{T}\left(v^{*}-u^{*}\right)=\left(\beta^{*}-\alpha^{*}\right)\left(u^{*}\right)^{T} v^{*} \geq\left(\beta^{*}-\alpha^{*}\right)\left\|u^{*}\right\|\left\|v^{*}\right\|=-\sigma\left(\left\|v^{*}\right\|-\left\|u^{*}\right\|\right)\left\|u^{*}\right\|\left\|v^{*}\right\|
$$

where we have used the Cauchy-Schwarz inequality (multiplied by $\beta^{*}-\alpha^{*}$ which is negative by (4.14)). We now have from (4.12) that

$$
\begin{aligned}
m^{-}\left(v^{*}\right)-m^{-}\left(u^{*}\right) & =\frac{1}{2} g^{T}\left(v^{*}-u^{*}\right)+\frac{\sigma}{6}\left(\left\|v^{*}\right\|^{3}-\left\|u^{*}\right\|^{3}\right) \\
& \geq-\frac{\sigma}{2}\left(\left\|v^{*}\right\|-\left\|u^{*}\right\|\right)\left\|u^{*}\right\|\left\|v^{*}\right\|+\frac{\sigma}{6}\left(\left\|v^{*}\right\|^{3}-\left\|u^{*}\right\|^{3}\right) \\
& =\frac{\sigma}{6}\left(-3\left\|v^{*}\right\|^{2}\left\|u^{*}\right\|+3\left\|u^{*}\right\|^{2}\left\|v^{*}\right\|+\left\|v^{*}\right\|^{3}-\left\|u^{*}\right\|^{3}\right) \\
& =\frac{\sigma}{6}\left(\left\|v^{*}\right\|-\left\|u^{*}\right\|\right)^{3} \geq 0
\end{aligned}
$$

which completes the proof. 


\section{A Faster Heuristic Algorithm}

It is not always necessary or desirable to find the global minimum to a high degree of accuracy. With this in mind we present a faster heuristic version of the Lipschitz based branch and bound algorithm which has no theoretical convergence guarantees but still exhibits reasonable performance.

The main drawback with regards to performance of the existing algorithm is the splitting of each ball into $3^{n}$ sub-balls since the number of sub-balls grows rapidly as $n$ (the dimension of the problem) increases. Rather than using overlapping balls, for the heuristic version of the algorithm we split each ball into a dense lattice of non-overlapping sub-balls. The maximum number of same-size balls one can pack around a central ball without overlap is given by the kissing number $\kappa$ (Conway and Sloane, 1999). Optimal kissing numbers and the corresponding lattices we use which give rise to them are known up to 9 dimensions (see Conway and Sloane, 1999, for details). Running the algorithm with this splitting rule means that each ball is only split into $\kappa+1$ sub-balls, considerably less than $3^{n}$ (cf. Table 1 ). The disadvantage is that it

\begin{tabular}{ccc}
\hline$n$ & $\kappa+1$ & $3^{n}$ \\
\hline 1 & 3 & 3 \\
2 & 7 & 9 \\
3 & 13 & 27 \\
4 & 25 & 81 \\
5 & 41 & 243 \\
6 & 73 & 729 \\
7 & 127 & 2187 \\
8 & 241 & 6561 \\
9 & 273 & 19683 \\
\hline
\end{tabular}

Table 1: The optimal kissing number plus one $(\kappa+1)$ compared against $3^{n}$ for the first 9 dimensions.

leaves holes in the domain we are trying to optimize over and so convergence to the global optimum is not guaranteed. However, by running a local solver from the global minimum proposed by the algorithm, we will always find a local minimum which is often a good candidate for being the global optimum (and which can be used as an upper bound in the original slower version of Algorithm 2.1 if desired). Figure 4 illustrates this heuristic splitting rule using the hexagonal lattice which is known to be the optimal lattice in two dimensions.

\section{Application to Radial Basis Functions}

In recent years radial basis function $(\mathrm{RBF})$ interpolation has become a popular and well established approximation method for real valued functions, particularly in higher dimensions (see Wendland, 2005). This approach is also known as intrinsic random function Kriging in the geostatistical literature (as proposed by Matheron, see Chilès and Delfiner, 1999) and Gaussian 


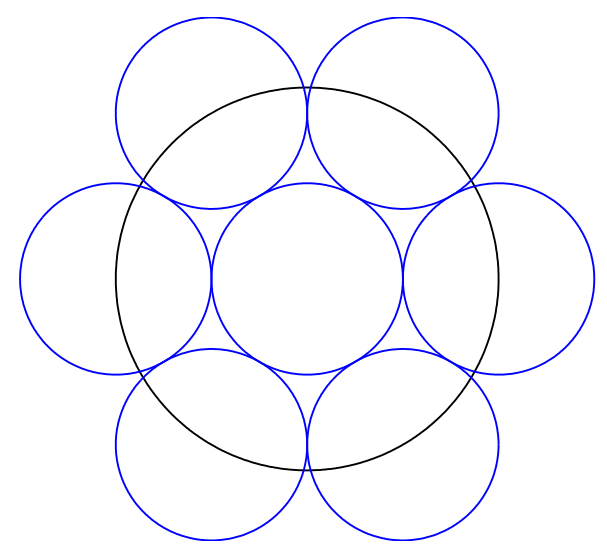

Figure 4: An illustration of our heuristic splitting rule in two dimensions. The black circle is split into seven blue circles arranged in a hexagonal lattice.

process regression in Bayesian statistics (an early reference is O'Hagan, 1978). Under these synonyms RBF interpolation has been applied to the design and analysis of computer experiments (Santner, Williams, and Notz, 2003), machine learning (Rasmussen and Williams, 2006) and engineering design (Forrester, Sóbester, and Keane, 2008) to name but a few. The RBF interpolant can also be viewed as a neural network (see Chapter 5 of Bishop, 1996). One of the main advantages of using RBFs to approximate an underlying real valued function is that one can cheaply obtain derivatives of the RBF of any order. In particular, this allows us to easily obtain the Hessian Lipschitz constant over any ball in $\mathbb{R}^{n}$ and thus apply our Lipschitz based branch and bound algorithm, Algorithm 2.1 from $\S 2$. Note that this is an entirely new approach, distinct from the canonical Lipschitz branch and bound algorithm (as described in e.g. Section 5.3 of Pardalos, Horst, and Thoai, 1995). With this in mind we will apply the proposed branch and bound algorithm to the special case where the objective function $f(x)$ is a radial basis function approximation to some real valued function. Of course, the real motivation behind using RBFs is to use the global minimizer of the RBF approximation to infer something about the original problem, i.e. the global minimum of the underlying real valued function that is approximated (see Farmer, Fowkes, and Gould, 2010, for an example application).

\subsection{Introduction to Radial Basis Functions}

First of all, let us give a brief introduction to radial basis function interpolation. We begin by defining the weighted $\ell_{2}$-norm $\|\cdot\|_{W}:=\|W \cdot\|_{2}$ with diagonal weight matrix $W$, and suppose we have $N$ samples $y=\left(y_{1}, \ldots, y_{N}\right)^{T}$ of some real valued function we wish to approximate at the corresponding sample points $x_{1}, \ldots, x_{N} \in \mathcal{D}$. An $\mathrm{RBF}$ approximation $f: \mathcal{D} \subset \mathbb{R}^{n} \rightarrow \mathbb{R}$ is then constructed as a linear combination of basis functions $\varphi(\cdot)$ composed with a weighted $\ell_{2}$-norm, together with an additional polynomial term to guarantee uniqueness:

$$
f(x)=\sum_{k=1}^{M} \mu_{k} \pi_{k}(x)+\sum_{j=1}^{N} \lambda_{j} \varphi\left(\left\|x-x_{j}\right\|_{W}\right)
$$


where $\left\{\pi_{k}(x)\right\}_{k=1}^{M}$ is a basis for $\Pi_{d}^{n}$, the space of polynomials in $\mathbb{R}^{n}$ of degree less than $d$, with the notation $\Pi_{0}^{n}=\{0\}$. Typical choices of the basis function $\varphi(\cdot)$ are

$$
\begin{aligned}
& \text { the spline } \quad \varphi(r)=\left\{\begin{array}{ll}
r^{p} & \text { if } p \text { is odd } \\
r^{p} \log r & \text { if } p \text { is even }
\end{array} ;\right. \\
& \text { the multiquadric } \quad \varphi(r)=\left(r^{2}+\gamma^{2}\right)^{\beta} \quad \beta>0, \beta \notin \mathbb{N} \text {; } \\
& \text { the inverse multiquadric } \quad \varphi(r)=\left(r^{2}+\gamma^{2}\right)^{-\beta} \quad \beta>0 \text {; and } \\
& \text { the Gaussian } \quad \varphi(r)=\exp \left(-\gamma^{2} r^{2}\right) \text {, }
\end{aligned}
$$

where $\gamma$ is a nonzero constant referred to as the shape parameter (see, for example, Chapters $6,7,8$ of Wendland, 2005). Note that spline type RBFs are not $C^{2}$ of $p=1$ nor $C^{3}$ unless $p$ is greater than two. As we use a weighted norm, we often let $\gamma=1$ for the Gaussian basis function. The coefficients $\mu_{k}, \lambda_{j}$ are determined by solving the linear interpolation system

$$
y_{i}=\sum_{k=1}^{M} \mu_{k} \pi_{k}\left(x_{i}\right)+\sum_{j=1}^{N} \lambda_{j} \varphi\left(\left\|x_{i}-x_{j}\right\|_{W}\right), \quad i=1, \ldots, N
$$

along with the additional conditions

$$
\sum_{j=1}^{N} \lambda_{j} \pi_{k}\left(x_{j}\right)=0, \quad k=1, \ldots, M
$$

which complete the system and ensure that polynomials of degree less than $d$ are interpolated exactly. In matrix form this gives the non-singular (provided $\left\{x_{i}\right\}_{i=1}^{N}$ is a $\prod_{d}^{n}$-unisolvent set, see Wendland, 2005) symmetric saddle-point system

$$
\left(\begin{array}{cc}
R & P \\
P^{T} & 0
\end{array}\right)\left(\begin{array}{l}
\lambda \\
\mu
\end{array}\right)=\left(\begin{array}{l}
y \\
0
\end{array}\right)
$$

where $P_{i, j}=\pi_{j}\left(x_{i}\right)$ is a polynomial basis matrix and $R$ is the correlation matrix given by

$$
R_{i, j}=\varphi\left(\left\|x_{i}-x_{j}\right\|_{W}\right) .
$$

There are two main approaches often used in the literature to find the weights in the weight matrix $W$, which we will now assume to be diagonal. The first approach consists of choosing $W$ to maximise the likelihood of the observed data $x_{1}, \ldots, x_{N}$ and leads one to choose $W$ to be the maximiser of the log-likelihood function (see Busby, Farmer, and Iske, 2007)

$$
\ell(W)=-\frac{1}{2}\left(N \log \sigma^{2}+\log \operatorname{det}(R)\right)
$$

which we optimise using a general purpose global optimisation algorithm (DIRECT, Jones, Perttunen, and Stuckman, 1993). The second approach is to use leave-one-out cross-validation (see Rippa, 1999) and leads one to choose $W$ to minimise the $\ell_{2}$-norm of the cross-validation error $\epsilon(W) \in \mathbb{R}^{N}$. The $k$-th element of the cross-validation error $\epsilon(W)$ is the error at the validation point $x_{k}$, given by

$$
\epsilon_{k}(W)=\frac{\lambda_{k}}{A_{k, k}^{-1}}
$$

where $A_{k, k}^{-1}$ is the $k$-th diagonal element of the inverse of the interpolation matrix $A=\left(\begin{array}{cc}R & P \\ P^{T} & 0\end{array}\right)$. 


\subsection{Calculating the Lipschitz constant}

We now proceed to calculating a suitable Hessian Lipschitz constant for our proposed branch and bound algorithm in the special case where the objective function $f(x)$ is a radial basis function approximation (6.1). We start by introducing tensors. A third order tensor $T$ is a generalisation of a matrix to three indices, that is to say a 3-dimensional array. As with matrices $T_{i, j, k}$ denotes the $i, j, k$-th component (i.e. element in the array) of the tensor $T$. Recall that the matrix Frobenius norm can be defined as

$$
\|A\|_{F}^{2}:=\sum_{i=1}^{m} \sum_{j=1}^{n} A_{i, j}^{2}
$$

and this can be extended to a third order tensor $T$ as

$$
\|T\|_{F}^{2}:=\sum_{i=1}^{m} \sum_{j=1}^{n} \sum_{k=1}^{o} T_{i, j, k}^{2} .
$$

Similarly, we can define the induced $\ell_{2}$-norm for tensors by

$$
\|T\|_{2}:=\max _{\|x\|_{2}=1}\|T x\|_{2}
$$

where $\|T x\|_{2}$ denotes the usual induced matrix norm. We are now in a position to prove the following Lemma.

Lemma 6.1. Let $T$ be a third order tensor. Then $\|T\|_{2} \leq\|T\|_{F}$.

Proof: We have that

$$
\begin{aligned}
\|T\|_{2}^{2} & =\max _{\|x\|_{2}=1}\|T x\|_{2}^{2} \leq \max _{\|x\|_{2}=1}\|T x\|_{F}^{2} \quad \text { as }\|A\|_{2} \leq\|A\|_{F} \text { for matrices } \\
& =\max _{\|x\|_{2}=1} \sum_{i=1}^{m} \sum_{j=1}^{n}\left(\sum_{k=1}^{o} T_{i, j, k} x_{k}\right)^{2} \\
& =\max _{\|x\|_{2}=1} \sum_{i=1}^{m} \sum_{j=1}^{n}\left(\sum_{k=1}^{o}\left(a_{i, j}\right)_{k} x_{k}\right)^{2} \text { where the vector } a_{i, j} \text { is such that }\left(a_{i, j}\right)_{k}=T_{i, j, k} \\
& \leq \max _{\|x\|_{2}=1} \sum_{i=1}^{m} \sum_{j=1}^{n}\left\|a_{i, j}\right\|_{2}^{2}\|x\|_{2}^{2} \quad \text { by the Cauchy-Schwarz inequality } \\
& =\sum_{i=1}^{m} \sum_{j=1}^{n}\left\|a_{i, j}\right\|_{2}^{2}=\sum_{i=1}^{m} \sum_{j=1}^{n} \sum_{k=1}^{o} T_{i, j, k}^{2}=\|T\|_{F}^{2}
\end{aligned}
$$

Let $T(x):=\nabla_{x x x} f(x)$ denote the third order derivative tensor of the RBF approximation $f(x)$ to some real valued function. We have from Taylor's theorem that for any $x, y \in \mathcal{B}$

$$
\|H(x)-H(y)\|_{2} \leq\left\|\int_{0}^{1} T(y+\tau(x-y))(x-y) d \tau\right\|_{2} \leq \max _{0 \leq \tau \leq 1}\|T(y+\tau(x-y))\|_{2}\|x-y\|_{2}
$$


where $\|T(\cdot)\|_{2}$ denotes the tensor $\ell_{2}$-norm defined above. Thus the Hessian $H(x):=\nabla_{x x} f(x)$ is Lipschitz continuous on a ball $\mathcal{B} \subset \mathbb{R}^{n}$ if there exists a $\ell_{2}$-norm Lipschitz constant $L_{H}(\mathcal{B})>0$ such that for all $x \in \mathcal{B}$

$$
\|T(x)\|_{2} \leq L_{H}(\mathcal{B}) .
$$

It suffices to find an upper bound $\tau(\mathcal{B})$ on $T(x)$ over $\mathcal{B}$ and we can then use Lemma 6.1 to calculate an upper bound $L_{H}(\mathcal{B})$ on the optimal Hessian Lipschitz constant as

$$
\|T(x)\|_{2} \leq\|\tau(\mathcal{B})\|_{2} \leq\|\tau(\mathcal{B})\|_{F}=\left(\sum_{i=1}^{m} \sum_{j=1}^{n} \sum_{k=1}^{o} \tau_{i, j, k}^{2}\right)^{1 / 2}=L_{H}(\mathcal{B}) .
$$

Thus it remains to determine the upper bound for the RBF approximation $f(x)$. The approximation $f(x)$ has the form (6.1)

$$
f(x)=\sum_{k=1}^{M} \mu_{k} \pi_{k}(x)+\sum_{j=1}^{N} \lambda_{j} \varphi\left(\left\|x-x_{j}\right\|_{W}\right)
$$

with associated third order derivative tensor $T(x)$ given by

$$
T(x)=\sum_{k=1}^{M} \mu_{k} \nabla_{x x x} \pi_{k}(x)+\sum_{j=1}^{N} \lambda_{j} \nabla_{x x x} \varphi\left(\left\|x-x_{j}\right\|_{W}\right) .
$$

To calculate the upper bound $\tau(\mathcal{B})$, it therefore suffices to calculate upper and lower bounds on the tensors $\nabla_{x x x} \pi_{k}(x)$ and $\nabla_{x x x} \varphi\left(\left\|x-x_{j}\right\|_{W}\right)$ over $\mathcal{B}$ depending on the signs of the coefficients $\lambda_{j}, \mu_{k}$. For example, for the cubic spline $\operatorname{RBF} \varphi(r)=r^{3}$ we have $\nabla_{x x x} \pi_{k}(x)=0$ as the polynomial term is linear and

$$
\left(\nabla_{x x x} \varphi\left(\left\|x-x_{j}\right\|_{W}\right)\right)_{a, b, c}= \begin{cases}\frac{-3 w_{a}^{6}\left(x_{a}-x_{j_{a}}\right)^{3}}{\left\|x-x_{j}\right\|_{W}^{3}}+\frac{9 w_{a}^{2}\left(x_{a}-x_{j_{a}}\right)}{\left\|x-x_{j}\right\|_{W}} & \text { if } a=b=c \\ \frac{-3 w_{c}^{2}\left(x_{c}-x_{j_{c}}\right) w_{a}^{4}\left(x_{a}-x_{j_{a}}\right)^{2}}{\left\|x-x_{j}\right\|_{W}^{3}}+\frac{3 w_{c}^{2}\left(x_{c}-x_{j_{c}}\right)}{\left\|x-x_{j}\right\|_{W}} & \text { if e.g. } a=b \neq c \\ \frac{-3 w_{a}^{2}\left(x_{a}-x_{j_{a}}\right) w_{b}^{2}\left(x_{b}-x_{j_{b}}\right) w_{c}^{2}\left(x_{c}-x_{j_{c}}\right)}{\left\|x-x_{j}\right\|_{W}^{3}} & \text { otherwise. }\end{cases}
$$

It is trivial to find upper and lower bounds on $w_{a}^{2}\left(x_{a}-x_{j_{a}}\right) /\left\|x-x_{j}\right\|_{W}$ over the smallest box containing $\mathcal{B}$ which we can substitute into the above to obtain bounds on $\nabla_{x x x} \varphi\left(\left\|x-x_{j}\right\|_{W}\right)$. A similar approach can be used for other radial basis functions (6.2) with interval arithmetic techniques for higher order polynomial terms.

\subsection{Numerical Examples}

To begin with let us look at an illustrative two dimensional example. Consider the problem of finding the global minimum of a cubic spline RBF approximation $f(x, y)$ to the Dixon-Szegó six hump camel back function at thirty scattered points in $[-2,2] \times[-1.25,1.25]$. We will use Algorithm 2.1 with overlapping balls and local Lipschitz constants from $\S 2$. The optimal solution as found in 244 iterations of Step 1 of the algorithm with a tolerance of $4 \times 10^{-6}$ is 
shown in Figure 5 below. This took about 25 seconds of cpu time for a Matlab implementation on an AMD Phenom II X3 705e processor machine.

Compare this with the heuristic version using a lattice of balls from $\S 5$. In this case, the optimal solution as found in 147 iterations of Step 1 of the algorithm with a tolerance of $6 \times 10^{-6}$ is shown in Figure 6 below. This took about 12 seconds of cpu time, considerably less. Notice how the alternate splitting rule is more efficient at filling the available space (albeit with gaps).

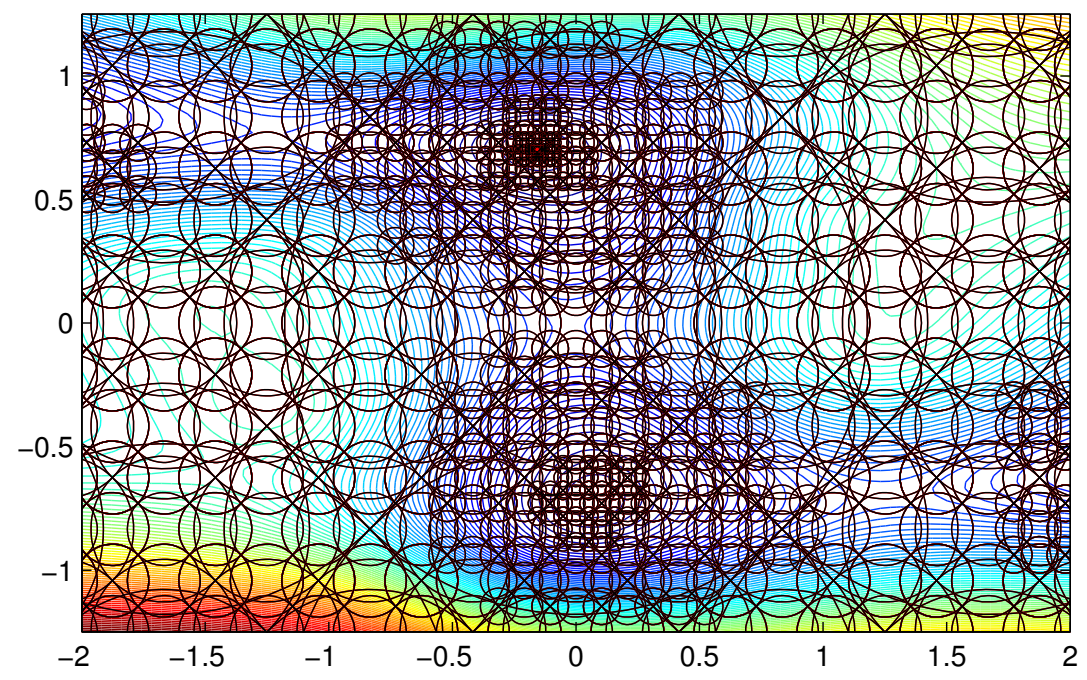

Figure 5: Contours of the RBF approximation $f(x, y)$ to the camel function. The black circles denote the overlapping closed balls $\mathcal{B}$ used by the branch and bound algorithm. Note that they cluster at the global minimum of $f(x, y)$ which is denoted by a red circle.

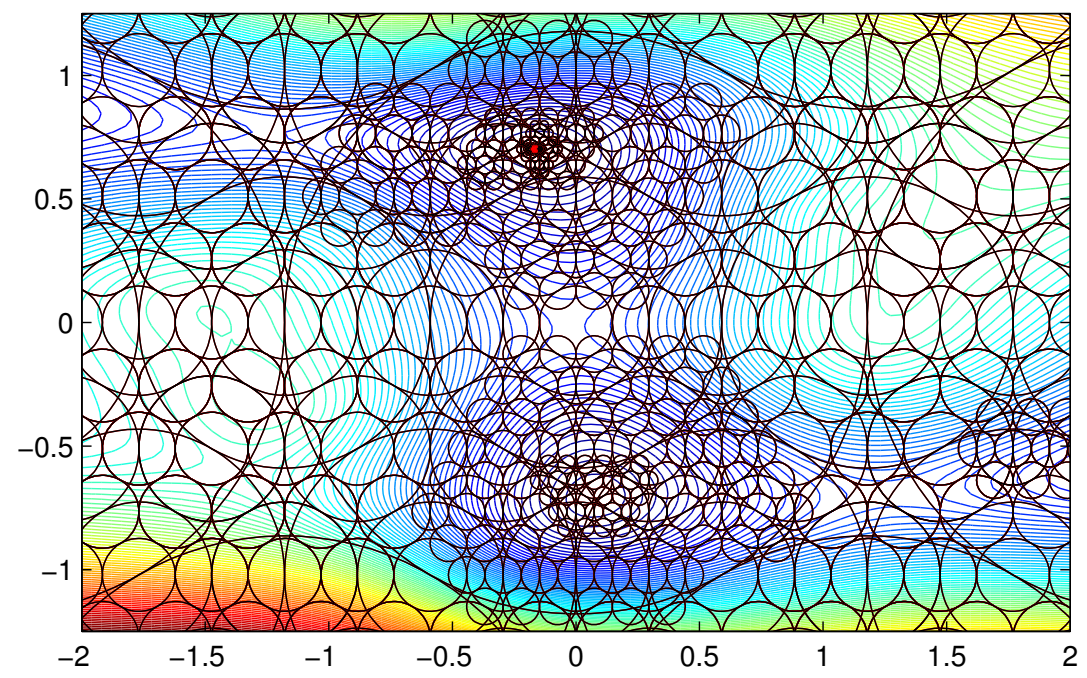

Figure 6: Contours of the RBF approximation $f(x, y)$ to the camel function. The black circles denote the hexagonal lattice of balls $\mathcal{B}$ used by the branch and bound algorithm. Note that they cluster at the global minimum of $f(x, y)$ which is denoted by a red circle.

For a more interesting example, we will now compare the performance of the two branch and bound algorithm variants for dimensions from 2 to 5 . In addition, we will also compare our 
algorithms to the canonical Lipschitz branch and bound algorithm (see Section 5.3 of Pardalos et al., 1995). This is simply the canonical branch and bound algorithm (see e.g. Horst, 1986) with the lower bound

$$
\alpha(\mathcal{B})=f\left(x_{\mathcal{B}}\right)-L(\mathcal{B}) \max _{x \in \mathcal{B}}\left\|x-x_{\mathcal{B}}\right\|
$$

where $x_{\mathcal{B}}$ is the midpoint of $\mathcal{B}$ and $L(\mathcal{B})$ an upper bound on the optimal Lipschitz constant over $\mathcal{B}$, calculated by bounding the norm of the gradient over $\mathcal{B}$ similarly to how we calculate $L_{H}(\mathcal{B})$ in $\S 6.2$. For this example, the objective function $f:[-4,4]^{n} \subset \mathbb{R}^{n} \rightarrow \mathbb{R}$ will be a cubic spline radial basis function approximation to the sum of sine functions given by

$$
s(x)=\sum_{k=1}^{n} \sin x_{k}
$$

at $10 n$ maximin Latin hypercube sample points in $[-4,4]^{n}$. The approximation typically has a number of local minima with one global minimum as one can see in Figure 7 for dimension $n=2$. Table 2 below shows the run time in seconds of a Matlab implementation of each branch and bound algorithm for dimensions $n$ from 2 to 5 . The algorithm was stopped if

\begin{tabular}{lcccc}
\hline & 2 & 3 & 4 & 5 \\
\hline Canonical Lipschitz $\left(L_{f}\right)$ & $145 \mathrm{~s}$ & $4 \times 10^{0} \dagger$ & $1 \times 10^{2} \dagger$ & $4 \times 10^{2} \dagger$ \\
Algorithm 2.1 $\left(L_{H}\right)$ & $4 \mathrm{~s}$ & $737 \mathrm{~s}$ & $3 \times 10^{1} \dagger$ & $4 \times 10^{3} \dagger$ \\
Heuristic Algorithm 2.1 $\left(L_{H}\right)$ & $\mathbf{3 s}$ & $\mathbf{8 1 s}$ & $\mathbf{2 8 8 6 s}$ & $\mathbf{4} \times \mathbf{1 0}^{\mathbf{1}} \dagger$ \\
\hline
\end{tabular}

Table 2: Run times of the different branch and bound algorithms on a radial basis function approximation to the sum of sines function for dimensions from 2 to 5 . The best results for each dimension are denoted in bold. †The tolerance reached is given instead if the algorithm did not complete in 50 minutes.

it verifiably found the global minimum to within a tolerance of $10^{-2}$, i.e. $U_{k}-L_{k}<10^{-2}$. If this was not possible in 3000 seconds (50 minutes) we give the tolerance reached by the algorithm instead. The experiments were performed on an AMD Phenom II X3 705e processor machine with 4 GB of RAM running Matlab R2010b and the NAG toolbox for Matlab, Mark 22 which provided the local optimization solvers. As one can see from the results, the canonical Lipschitz branch and bound algorithm shows the worst performance and takes considerably more iterations than all the other algorithms. This is because the lower bounding function (6.4) used in the algorithm only makes use of the function Lipschitz constant and is therefore quite crude. The other algorithms use much tighter bounds which explains their superior performance in lower dimensions. As the dimension increases, the need to split $3^{n}$ balls at each iteration hampers the performance of our Lipschitz algorithm, however the heuristic version (which splits considerably fewer balls at each iteration) consistently outperforms the overlapping balls algorithm. Nonetheless, one must concede that the tolerance returned by the heuristic algorithm is often that of a global minimum on a subset of $[-4,4]^{n}$, particularly in higher dimensions. While the inability of the algorithms to complete in higher dimensions may seem disappointing one must bear in mind that these are research implementations written in object oriented 

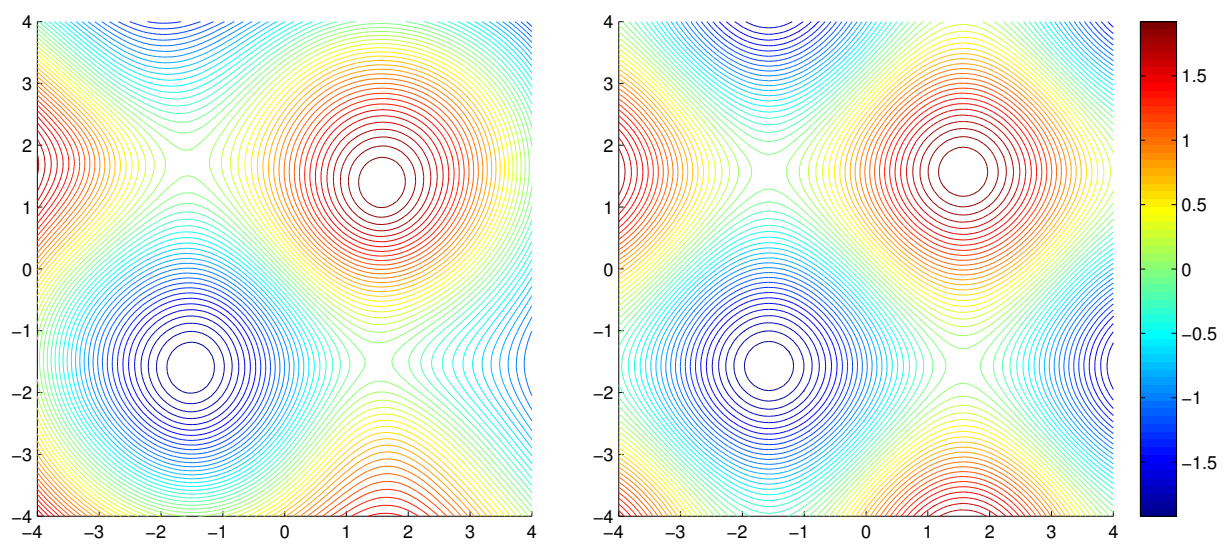

Figure 7: Contours of the radial basis function approximation (left) to the sum of sines function (right) on $[-4,4] \times[-4,4]$. As one can see the approximation is particularly accurate for this example.

Matlab which is very slow. Moreover, our Lipschitz algorithm can be trivially parallelised as the bounds on each ball can be computed independently which should lead to a significant speedup on modern multiprocessor hardware.

Let us now compare the our proposed branch and bound algorithm with overlapping balls and its heuristic counterpart subject to convex constraints. As in the previous example, the objective function $f:[-4,4]^{n} \subset \mathbb{R}^{n} \rightarrow \mathbb{R}$ will be a cubic spline radial basis function approximation to the sum of sine functions, this time with the elliptical constraint

$$
c(x)=x^{T} C x-1 \leq 0
$$

where $C$ is a matrix with $1 / 2$ on the diagonal and $1 / 4$ elsewhere. This time the global minimum of the constrained surrogate typically lies on the boundary as one can see in Figure 8. It is our intention to test the convergence of the algorithms on the boundary, for if the global minimum were to lie in the interior, performance would be comparable to the bound constrained example above. Table 3 below shows the run time in seconds of a Matlab implementation of each

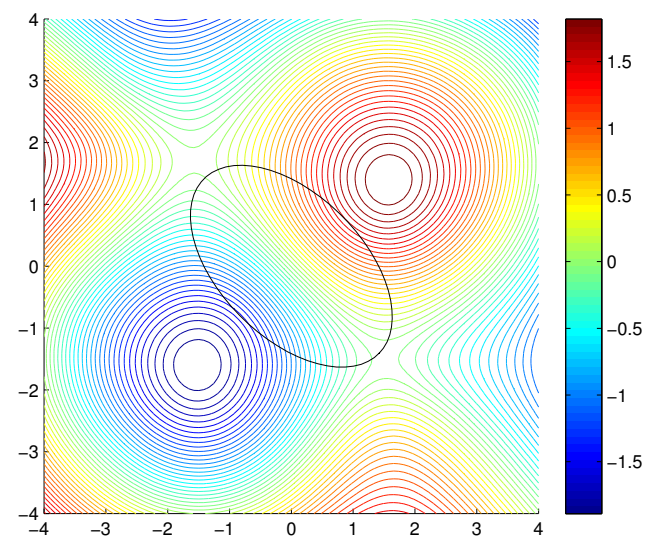

Figure 8: Contours of the radial basis function approximation to the sum of sines function on $[-4,4] \times[-4,4]$ with the elliptical constraint region denoted in black.

constrained branch and bound algorithm for dimensions $n$ from 2 to 5 . The testing methodology 
and hardware is the same as in the previous experiments. The speed advantage of the heuristic

\begin{tabular}{lcccc}
\hline & 2 & 3 & 4 & 5 \\
\hline Algorithm 2.1 $\left(L_{H}\right)$ & $4 \mathrm{~s}$ & $5 \times 10^{-2} \dagger$ & $1 \times 10^{1} \dagger$ & $3 \times 10^{1} \dagger$ \\
Heuristic Algorithm 2.1 $\left(L_{H}\right)$ & $5 \mathrm{~s}$ & $\mathbf{4 8 5 \mathrm { s }}$ & $\mathbf{2 \times \mathbf { 1 0 } ^ { - \mathbf { 2 } } \dagger}$ & $\mathbf{1 \times 1 0 ^ { - \mathbf { 1 } } \dagger}$ \\
\hline
\end{tabular}

Table 3: Run times of the different convex constrained branch and bound algorithms on a radial basis function approximation to the sum of sines function for dimensions from 2 to 5. The best results for each dimension are denoted in bold. $\dagger$ The tolerance reached is given instead if the algorithm did not complete in 50 minutes.

Lipschitz algorithm is evident, once again it consistently outperforms the algorithm using balls for dimensions greater than 2. Even so, the results are worse than in the bound constrained example above. This is because the boundary contains a large region where the function takes similar values and the algorithms expend a significant effort searching this region for the global minimiser. However, in such situations a constrained local search algorithm can easily locate the global minimum once the branch and bound algorithm has located its basin of attraction.

Finally, let us consider the performance of Algorithm 2.1 and its heuristic counterpart on RBF approximations to the extended Dixon-Szegó test set (see Dixon and Szegó, 1978) which is widely used in the global optimization literature (see e.g. Huyer and Neumaier, 2008). The test set consists of nine functions with dimensions $n$ ranging from two to six all defined on rectangular domains. Table 4 below gives a brief overview of the test functions and Table 5 gives results for the algorithms on RBF approximations to the test functions. These approximations interpolate each test function at $10 n$ Halton samples (Halton, 1960). The testing methodology and hardware is the same as for the previous examples and we once again compare against the canonical Lipschitz branch and bound algorithm.

\begin{tabular}{lcccc}
\hline & $n$ & Local Minima & Global Minima & $\mathcal{D}$ \\
\hline Branin & 2 & 3 & 3 & {$[-5,10] \times[0,15]$} \\
Six-hump Camel & 2 & 6 & 2 & {$[-3,3] \times[-2,2]$} \\
Goldstein-Price & 2 & 4 & 1 & {$[-2,2]^{2}$} \\
Shubert & 2 & 760 & 18 & {$[-10,10]^{2}$} \\
Hartman 3 & 3 & 4 & 1 & {$[0,1]^{3}$} \\
Shekel 5 & 4 & 5 & 1 & {$[0,10]^{4}$} \\
Shekel 7 & 4 & 7 & 1 & {$[0,10]^{4}$} \\
Shekel 10 & 4 & 10 & 1 & {$[0,10]^{4}$} \\
Hartman 6 & 6 & 4 & 1 & {$[0,1]^{6}$} \\
\hline
\end{tabular}

Table 4: The dimension $n$, number of local and global minima and domain $\mathcal{D}$ for each of the Dixon-Szegö test functions. 


\begin{tabular}{|c|c|c|c|}
\hline $\begin{array}{c}\mathrm{RBF} \\
\text { approximation to }\end{array}$ & Algorithm $2.1\left(L_{H}\right)$ & $\begin{array}{c}\text { Heuristic } \\
\text { Algorithm 2.1 }\left(L_{H}\right)\end{array}$ & $\begin{array}{c}\text { Canonical } \\
\text { Lipschitz }\left(L_{f}\right)\end{array}$ \\
\hline Branin & $7 \mathrm{~s}$ & $5 \mathrm{~s}$ & $78 \mathrm{~s}$ \\
\hline Six-hump Camel & $19 \mathrm{~s}$ & $9 \mathrm{~s}$ & $4 \times 10^{-2} \dagger$ \\
\hline Goldstein-Price & $144 \mathrm{~s}$ & $203 \mathrm{~s}$ & $4 \times 10^{3} \dagger$ \\
\hline Shubert & $18 \mathrm{~s}$ & $15 \mathrm{~s}$ & $2 \times 10^{2} \dagger$ \\
\hline Hartman 3 & $562 \mathrm{~s}$ & $72 \mathrm{~s}$ & $5 \times 10^{0} \dagger$ \\
\hline Shekel 5 & $1 \times 10^{1} \dagger$ & $9 \times 10^{-2} \dagger$ & $2 \times 10^{0} \dagger$ \\
\hline Shekel 7 & $7 \times 10^{0} \dagger$ & $1067 \mathrm{~s}$ & $4 \times 10^{0} \dagger$ \\
\hline Shekel 10 & $7 \times 10^{0} \dagger$ & $1076 \mathrm{~s}$ & $4 \times 10^{0} \dagger$ \\
\hline Hartman 6 & $1 \times 10^{3} \dagger$ & $1 \times 10^{2} \dagger$ & $1 \times 10^{2} \dagger$ \\
\hline
\end{tabular}

Table 5: Run times (in seconds) of the two variants of Algorithm 2.1 and the canonical Lipschitz algorithm on a radial basis function approximation to functions from the DixonSzegó test set. The best results for each dimension are denoted in bold. $\dagger$ The tolerance reached is given instead if the algorithm did not complete in 50 minutes.

\section{Conclusions}

We have presented an entirely new algorithm for the minimisation of of a twice differentiable nonconvex objective function with a Lipschitz continuous Hessian over a compact, convex set. The algorithm is based on the canonical branch and bound global optimization algorithm with bounds inspired by the trust region subproblem from local optimization. Numerical results suggest that the proposed algorithm outperforms existing Lipschitz based approaches in the literature when applied to a radial basis function approximation and one would expect good performance in other settings where a Hessian Lipschitz constant is available. One main advantage of the proposed algorithm is that it is naturally parallelisable, especially in higher dimensions, and we are currently looking into a large-scale parallel implementation.

\section{Acknowledgements}

We would like to thank Coralia Cartis for helpful suggestions on an early draft of this paper.

\section{References}

I. P. Androulakis, C. D. Maranas, and C. A. Floudas. $\alpha$ BB: A Global Optimization Method for General Constrained Nonconvex Problems. Journal of Global Optimization, 7:337-363, 1995. http://dx.doi.org/10.1007/BF01099647.

V. Balakrishnan, S. Boyd, and S. Balemi. Branch and Bound Algorithm for Computing the Minimum Stability Degree of Parameter-Dependent Linear Systems. International Journal of Robust and Nonlinear Control, 1(4):295-317, 1991. http://dx.doi.org/10.1002/ rnc.4590010404. 
C. M. Bishop. Neural Networks for Pattern Recognition. Oxford University Press, 1996. ISBN 978-0-19-853864-6. URL http://books.google.co.uk/books?id=-aAwQO_-rXwC.

S. Boyd and L. Vandenberghe. Convex Optimization. Cambridge University Press, 2004. ISBN 978-0-521-83378-3. URL http://www.stanford.edu/ boyd/cvxbook/.

D. Busby, C. L. Farmer, and A. Iske. Hierarchical Nonlinear Approximation for Experimental Design and Statistical Data Fitting. SIAM Journal on Scientific Computing, 29(1):49-69, 2007. http://dx.doi.org/10.1137/050639983.

C. Cartis, N. I. M. Gould, and Ph. L. Toint. Adaptive Cubic Regularisation Methods for Unconstrained Optimization. Part I: Motivation, Convergence and Numerical Results. Mathematical Programming, 2009. http://dx.doi.org/10.1007/s10107-009-0286-5.

J.P. Chilès and P. Delfiner. Geostatistics: Modeling Spatial Uncertainty. Wiley Series in Probability and Statistics. Wiley, 1999. ISBN 978-0-471-08315-3. URL http://books.google.co. uk/books?id=adkSAQAAIAAJ.

A. R. Conn, N. I. M. Gould, and Ph. L. Toint. Trust Region Methods. MPS-SIAM Series on Optimization. SIAM, 2000. ISBN 978-0-89871-460-9. URL http://books.google.co.uk/ books?id=5kNC4fqssYQC.

J.H. Conway and N.J.A. Sloane. Sphere Packings, Lattices, and Groups, volume 290 of Grundlehren der mathematischen Wissenschaften. Springer, 1999. ISBN 978-0-387-98585-5. URL http://www.springer.com/mathematics/algebra/book/978-0-387-98585-5.

L. C. W. Dixon and G. P. Szegó. The Global Optimisation Problem: An Introduction. In L. C. W. Dixon and G. P. Szegó, editors, Towards Global Optimisation 2, pages 1-15. North-Holland, 1978. ISBN 978-0-444-85171-0. URL http://books.google.co.uk/books?id= SUSrAAAAIAAJ.

C. L. Farmer, J. M. Fowkes, and N. I. M. Gould. Optimal Well Placement. Presented at the 12th European Conference on the Mathematics of Oil Recovery, Oxford, 6 - 9 September, 2010. URL http://www.earthdoc.org/detail.php?pubid $=41313$.

C.A. Floudas and P.M. Pardalos. Handbook of Test Problems in Local and Global Optimization. Nonconvex optimization and its applications. Kluwer, 1999. ISBN 978-0-7923-5801-5. URL http://books.google.co.uk/books?id=vndwQgAACAAJ.

A.I.J. Forrester, A. Sóbester, and A.J. Keane. Engineering Design via Surrogate Modelling: A Practical Guide. Progress in Astronautics and Aeronautics. Wiley, 2008. ISBN 978-0-47006068-1. URL http://books.google.co.uk/books?id=AukeAQAAIAAJ.

N. I. M. Gould, D. P. Robinson, and H. S. Thorne. On Solving Trust-Region and Other Regularised Subproblems in Optimization. Mathematical Programming Computation, 2(1): 21-57, 2010. http://dx.doi.org/10.1007/s12532-010-0011-7. 
A. Griewank. The Modification of Newton's Method for Unconstrained Optimization by Bounding Cubic Terms. Technical Report NA/12 (1981), Department of Applied Mathematics and Theoretical Physics, University of Cambridge, 1981.

J. H. Halton. On the Efficiency of Certain Quasi-Random Sequences of Points in Evaluating Multi-Dimensional Integrals. Numerische Mathematik, 2:84-90, 1960. http://dx.doi.org/10. $1007 / \mathrm{BF} 01386213$.

R. Horst. A General Class of Branch-and-Bound Methods in Global Optimization with Some New Approaches for Concave Minimization. Journal of Optimization Theory and Applications, 51(2):271-291, 1986. http://dx.doi.org/10.1007/BF00939825.

R. Horst and P. M. Pardalos. Handbook of Global Optimization, volume 2 of Nonconvex Optimization and its Applications. Springer, 1995. ISBN 978-0-7923-3120-9. URL http://www.springer.com/mathematics/book/978-0-7923-3120-9.

Waltraud Huyer and Arnold Neumaier. SNOBFIT - Stable Noisy Optimization by Branch and Fit. ACM Transactions on Mathematical Software, 35(2):9:1-9:25, 2008. http://dx.doi. org/10.1145/1377612.1377613.

D. R. Jones. A Taxonomy of Global Optimization Methods Based on Response Surfaces. Journal of Global Optimization, 21(4):345-383, 2001. http://dx.doi.org/10.1023/A: 1012771025575.

D. R. Jones, C. D. Perttunen, and B. E. Stuckman. Lipschitzian Optimization Without the Lipschitz Constant. Journal of Optimization Theory and Applications, 79(1):157-181, 1993. http://dx.doi.org/10.1007/BF00941892.

Vladik Kreinovich and R. Kearfott. Beyond Convex? Global Optimization is Feasible Only for Convex Objective Functions: A Theorem. Journal of Global Optimization, 33:617-624, 2005. http://dx.doi.org/10.1007/s10898-004-2120-1.

Yurii Nesterov and B.T. Polyak. Cubic Regularization of Newton Method and its Global Performance. Mathematical Programming, 108(1):177-205, 2006. http://dx.doi.org/10.1007/ s10107-006-0706-8.

A. Neumaier. Complete Search in Continuous Global Optimization and Constraint Satisfaction. Acta Numerica, 13:271-369, 2004. http://dx.doi.org/10.1017/S0962492904000194.

A O'Hagan. Curve Fitting and Optimal Design for Prediction. Journal of the Royal Statistical Society B, 40(1):1-42, 1978. http://dx.doi.org/10.2307/2984861.

P. M. Pardalos and H. E. Romeijn. Handbook of Global Optimization Volume 2, volume 62 of Nonconvex Optimization and its Applications. Springer, 2002. ISBN 978-1-4020-0632-6. URL http://www.springer.com/mathematics/book/978-1-4020-0632-6. 
P. M. Pardalos, R. Horst, and N. V. Thoai. Introduction To Global Optimization, volume 3 of Nonconvex Optimization and its Applications. Springer, 1995. ISBN 978-0-7923-3556-6. URL http://www.springer.com/mathematics/book/978-0-7923-3556-6.

C. E. Rasmussen and C. K. I. Williams. Gaussian Processes for Machine Learning. Adaptive Computation and Machine Learning. MIT Press, 2006. ISBN 978-0-262-18253-9. URL http://www.gaussianprocess.org/gpml/chapters/RW.pdf.

S. Rippa. An Algorithm for Selecting a Good Value for the Parameter c in Radial Basis Function Interpolation. Advances in Computational Mathematics, 11(2):193-210, 1999. http: //dx.doi.org/10.1023/A:1018975909870.

T. J. Santner, B. J. Williams, and W. Notz. The Design and Analysis of Computer Experiments. Springer Series in Statistics. Springer, 2003. ISBN 978-0-387-95420-2. URL http://www. springer.com/statistics/statistical+theory + and + methods/book/978-0-387-95420-2.

J. C. Spall. Introduction to Stochastic Search and Optimization. Wiley-Interscience Series in Discrete Mathematics and Optimization. Wiley, 2003. ISBN 978-0-471-33052-3. URL http://books.google.co.uk/books?id=f66OIvvkKnAC.

H. Wendland. Scattered Data Approximation. Cambridge Monographs on Applied and Computational Mathematics. Cambridge University Press, 2005. ISBN 978-0-521-84335-5. URL http://books.google.co.uk/books?id=qy4cbWUmSyYC. 